\title{
China: A Shift from One-Child Policy to No-Child Policy
}

\author{
Jiawang Zhang ${ }^{1, *}$ \\ ${ }^{1}$ New York University, MS in Global Affairs, New York City, NY 10013, America \\ ${ }^{*}$ Corresponding author. Email: jz2362@outlook.com
}

\begin{abstract}
This research study aims at exploring the effects that have been experienced because of the one-child policy; whether the two-child policy is a good or bad alternative of one-child policy; as well as factors that influence individuals preferences of having a second-child. The effects will be critically analyzed in order to fully comprehend the extent to which the policy has affected local Chinese populations, in general. Study uses survey and interview. Study survey participants included 20 participants aged from 25 to 44 years' old who have the knowledge of the Chinese one-child policy. The six interviewees were chosen from the 20 participants aged from 27 to 32 years old. Survey results showed that a good percentage of participants recognized the positive consequences. Specifically, $85 \%$ of people that participated in the online survey indicated that the one-child policy promoted the attainment of quality education by Chinese people. Alternatives to the one-child policy do not raise hopes in the Chinese people according to the responses provided by participants. Specifically, a total of $40 \%$ of participants strongly agreed and agreed that the two-child policy was a good alternative to the one-child policy. Interview results showed that the main factors of having a second child are childhood experience, marital relationship and resources which influenced individual's fertility preferences.

The one-child policy was developed with the intent to benefit the Chinese people. The two-child policy is a good alternative for one-child policy. The childhood experience, marital relationship and resources have become the main factors for people to having a second child.
\end{abstract}

Keywords: One-child Policy, two-child policy alternative, factors, childhood experiences.

\section{INTRODUCTION}

The population of the People's Republic of China has always experienced massive growth trends. When China was founded in 1949 , the country had a population of approximately 540 million people; this population then doubled to reach approximately 830 million in the late 1970s (Ke et al., 2016). Initially, the high population growth was not considered any threat mainly because Mao Zedong, the Communist Party leader, believed that such a population growth would benefit the country's economy and power (Howden \& Zhou, 2014). However, the country's' population continued to experience growth at an unprecedented rate in the following decades. This rapid growth created fear in the country's government because of the potential constraints in natural, social, and economic resources. As a result, China has always sought to devise ways to control the country's population.
In 1984, the one-child policy was passed into law and any couple who was found to have exceeded the recommended number of children was penalized with sanctions that were both administrative and economic in nature.

Ultimately, parents with one child always felt the pressure. This trend was reflected by the desire to push the child to achieve high levels of success since there was no other sibling for parents to place their hopes (Falbo, 2018). In the behavioral context, families that had only one child were more reluctant to punish them in an effort to mold their behavior in comparison to families that had more children. Eventually, it could have an effect on the type of children that grew in a one-child home and those who came from families with at least two children. In this case, the one-child policy not only had an effect on the social and economic environment but also influenced the family context in terms of the structure and parenting 
styles adopted by couples who had an only child and those who had at least two children.

The rapid population growth in China is still a major concern. Most recently, the Chinese government has presented an alternative approach, namely the two-child policy, which was introduced in 2016 (Liu \& Liu 2017). Under this policy, Chinese couples can now have two children regardless of their ethnic background or whether they live in rural or urban areas of China. The main rationale behind the development and implementation of the new policy was the need to address the growing population of the older Chinese individuals and disproportion in age composition. The introduction of the two-child policy will not have any adverse effects such as rapid and unprecedented population growth of China's population to the one experienced before the enactment of the one-child policy. The one-child policy has left behind a lot of social problems, and has subtly changed people's view on marriage and reproduction.

This research study aims at exploring the effects that have been experienced because of the one-child policy; whether the two-child policy is a good or bad alternative of one-child policy; as well as factors that influence individuals' preferences of having a second-child. The effects will be critically analyzed in order to fully comprehend the extent to which the policy has affected local Chinese populations, in general.

\subsection{Methods}

The current study combined a quantitative and qualitative research design. This study uses a quantitative research design. According to the description provided by Albers, this design is effective in drawing meaning from a set of data gathered with the help of nonexperimental means, for example, surveys (Albers, 2017). As a result, the current study employs this design mainly because of the use of statistical data, which provide the opportunity to stay objective. In addition, by making use of statistical data, which were gathered by the use of quantitative research design, the researcher managed to develop a general perspective on various elements of China's child policy. In such a manner, the findings of the current study can be replicated by other researchers that aim to assess the policy from own perspectives. In addition, the researcher used the interview for understanding the factors of having the second child under the law of Chinese two-child policy.

\subsection{Survey participants and interviewee}

The topic required that the participants included in the study to have been affected by the one-child policy in one way or another as well as to know the intricate details of the policy. As a result, participants consisted of a number of individuals with different characteristics, including business people and policymakers from China. A total of 20 participants were involved in the survey process. These 20 participants also differed in demographic variables. To make sure that all participants involved in the study were given a fair chance to participate, a simple random sampling approach was used as the sampling technique. Another major advantage of using this sampling technique was the fact that the process had to be easily understood both by the researcher and the intended audience. Additionally, results obtained through this sampling technique can be projected, thus enabling the researcher to access the necessary materials for the process in proper time. The rationale for including individuals of varying demographics was to allow the researcher to obtain responses from representatives of different groups of people who had different perspectives. (See Table 1 below).

Table 1. Gender, Age and Nationality of participants

\begin{tabular}{|c|c|c|}
\hline Characteristics & Frequency & Percentage $(\%)$ \\
\hline \multicolumn{3}{|l|}{ Gender } \\
\hline Male & 12 & 60 \\
\hline Female & 8 & 40 \\
\hline \multicolumn{3}{|l|}{ Age } \\
\hline 25-34 years & 3 & 15 \\
\hline $35-44$ years & 10 & 50 \\
\hline Over 44 years & 7 & 35 \\
\hline \multicolumn{3}{|l|}{ Nationality } \\
\hline Chinese & 18 & 90 \\
\hline Non-Chinese & 2 & 10 \\
\hline Total & 20 & 100 \\
\hline
\end{tabular}

To make sure that all participants involved in the study were given a fair chance to participate, a simple random sampling approach was used as the sampling technique. Another major advantage of using this sampling technique was the fact that the process had to be easily understood both by the researcher and the intended audience (Taherdoost, 2016). Additionally, results obtained through this sampling technique can be projected, thus enabling the researcher to access the necessary materials for the process in proper time. The 
six interviewees were chosen from the 20 participants of the survey. (See Table 2 below).

Table 2. Characteristics of Interviewees

\begin{tabular}{l|cllllll}
\hline Characteristics & Age & Gender & Marital & Number of & Number of & Living & Location \\
& & & Status & Children & Siblings & Arrangement & \\
\hline Interviewee 1 & 27 & Male & Single & 0 & 1 & Living Alone & Beijing \\
\hline Interviewee 2 & 28 & Male & Married & 1 & 0 & With Partner & Yuyao \\
\hline Interviewee 3 & 27 & Female & Married & 0 & 0 & Living Alone & Shanghai \\
\hline Interviewee 4 & 27 & Female & Single & 0 & 1 & With Partner & Hohhot \\
\hline Interviewee 5 & 32 & Female & Married & 1 & 0 & With Partner & Dalian \\
\hline Interviewee 6 & 30 & Male & Single & 0 & 0 & Living Alone & Hohhot \\
\hline
\end{tabular}

\section{RESULT}

\subsection{Data Analysis}

All survey questionnaires distributed to participants were filled in and sent back to the researcher for further analysis. Hence, the participation and response rate based on the online survey was $100 \%$.

\subsection{Demographics of Participants}

The majority of participants (12 participants) were males. There were three participants aged 25-34 years, ten participants aged 35-44 years, seven participants aged over 44 years. The majority of participants (18 participants) were Chinese.

\subsection{Results of the Survey on China's One- Child Policy}

This section of the results was based on a 5-point Likert scale. In such a manner, participants were given 10 questions answer. The answers have been summarized into five parts.

\subsubsection{Knowledge about the history of China's one-child policy}

The majority of participants (15 participants) indicated that they have the adequate knowledge about the history of China's one-child policy. Five participants indicated that they have the knowledge of China's onechild policy.

\subsubsection{The role of one-child policy in wellbeing}

Most participants (12 participants) strongly agreed that the one-child policy contributed to the improvement of children's wellbeing. Six participants agreed and two participants remained neutral.
The majority of participants (17 participants) strongly agree and three participants agree that the onechild policy promoted the attainment of quality education.

One of the major concerns of the Chinese government in implementing the one-child policy was the constraint on resources. This concern was also reflected at the household level based on the ability of parents to cater to the needs and overall wellbeing of a large number of children adequately. As a result, the onechild policy contributed to a tradeoff, involving a number of children that individuals had and the associated quality of children's life. This tradeoff is in line with the quantity-quality trade-off theory developed by Gary Becker (Doepke, 2015).

\subsubsection{The role of one-child policy in gender equality}

Six participants strongly agreed that China experienced improvement in gender equality as a result of the on-child policy, four participants agreed. Five participants disagreed with the statement, and the remaining five strongly disagreed.

Participants gave equally positive and negative responses concerning the role of China's one-child policy in promoting gender inequality in the country. Five participants strongly agreed with this statement, while the other five participants agreed. On the other hand, six participants indicated that they disagreed with the statement, and four participants strongly disagreed.

The majority of participants (13 participants) strongly agreed and seven participants agreed that the one-child policy led to an increase in the male population and a decrease in the female population.

The influence of the one-child policy on gender ratio was twofold. On one hand, the policy led to the promotion of gender equality; on the other hand, it 
contributed to gender inequality in China. First, Sudbeck establishes that females born as the only children benefited greatly from the policy (Sudbeck, 2012). Based on the quality-quantity tradeoff theory, parents who cherished their female children rather than pondering on the lack of a male child invested significantly into the female offspring. Girls who were the only child were able to obtain better opportunities, in terms of education, political positions, social standing, and general rights and empowerment.

\subsubsection{The role of one-child policy in population size and structure.}

A large number of participants (12 participants) strongly agreed that the one-child policy was effective in controlling the Chinese population. Two participants were neutral; four participants disagreed and two participants strongly disagreed with the statement.

Most participants (14 participants) strongly agreed and six participants agreed that China's one-child policy led to a decrease in China's young population received positive responses from all participants.

The majority of participants (17 participants) strongly agreed and three participants agreed that China's aging population to the implementation of the one-child policy. China has an elderly insurance scheme in place that was developed in 2009 known as the New Rural Old Age Insurance Program (Zeng \& Hesketh, 2016). Despite its implementation, most elderly people in China still do not have complete pension coverage to support themselves financially. As a result, a large number of the elderly depend solely on the children in the family for financial support. One challenging factor is that the health needs of the elderly are immense. Most elderly people suffer from a wide range of age-associated diseases, disabilities, and dementia that pose a major challenge to the health care system and adult children, in general.

\subsubsection{The two-child policy as alternative to the one-child policy}

Five participants strongly agreed that the two-child policy is a good alternative to the one-child policy in China, three participants agreed. Six participants remained neutral. Four participants disagreed with the statement, while two participants strongly disagreed.

\subsection{Results of the Interviews on China's Two- Child Policy}

\subsubsection{Whether the National Policy Has Any Infuence on the Individual's Fertility Desire.}

Five participants suggested that the implementation of the two-child policy is mainly to solve the problem of
China's aging population. For example, Participant 1 said "My first thought was whether it was because of China's demographic structure. Now that it appears to be aging and tends to age, the birth rate needs to be improved." Participant 2 said "I think the implementation of the second child policy is inevitable, because lots of my friends do not want to have a child or have vary late birth, that fewer and fewer children lead to a serious problem of population aging. " Participant 5 said "this is a policy might be going to cope with an aging population and low birth rate. In order to resolve these two demographic issues. "

participant 5 put forward, "the demographic dividend is declining, as well as the pressure of social pensions, the burden is heavy. So in this case, the shortcomings of the one-child policy are beginning to show up, and then the country will change its population policy at this time. "

Participant 6 put forward the inertia of the one-child policy "because our generation are all only children, so we are actually used to it. There is not much the concept of having several children in the family." In this interview, participants generally believed that China's implementation of the two-child policy was to solve the relevant social problems left over from the one-child policy, the inertia of the one-child policy was affected by the interviewee's deep-rooted concept of the one-child policy.

\subsubsection{Micro: What are the Factors that affect Individual Fertility?}

The six participants all pointed out that the main determinant of choosing have a second child lies in personal willingness rather than national policies. Common factors of having the second child included childhood experience, marital relationship and social economic resources.

\subsubsection{Childhood Experience:}

Most participants are willing to have the second child based on their childhood experience. Participants 2, 3, 4 are the only-child in their family and willing to give birth to the second child. The reason is their childhood experience, they hope their children will be accompanied by their brothers and sisters in their future lives. Participant 2 said "I would love to have at least two children, so that they can accompany with each other, grow up together and take care of each other." Participant 3 said "I have a plan to have a second child. I think the main reason for having two children is my family will be more lively. " Participant 4 said " I am the only-child of my family, I felt lonely sometimes so I would love to have the second child." Participant 1 has a younger brother in his family because his family is Chinese ethnic minorities. Based on the 
influence of his childhood experience, he believes that one more child in the family will give him accompany. It is normal for him to have a second child.

Two participants hold a neutral stand on having the second child based on their childhood experience. Participant 6 said he has accepted this single family model, so he is unwilling to change. Participant 5 also has a younger brother in her family, she feels that her parents' love has been shared, so she maintained neutral about having a second child

\subsubsection{Marital Relationship}

Participants 5 and 6 both pointed out the intimacy of the marital relationship is a significant factor for having the second child. Participant 5 said " I think the decisive factor in having a child is the relationship between you and your partner. It depends on the relationship stability between you and your significant other, and whether the two of you can accept having a child in an intimate relationship." Participant 6 said "one of the most important factor of having the second child is the intimacy of the marital relationship. "

\subsubsection{Resources}

Participants 1, 5, 6 all pointed out that resources have become one of the important considerations for having a second child. Participant 1 said "I personally feel that the willingness to have children lies more in a series of cost issues such as the economy, education, and resources of raising children." Participant 5 raised economic factors by saying that "the economic ability of both husband and wife is one of the factors I consider to have a second child. " Prticipant 6 said "the competitiveness of the society and the economic environment are my considerations of having the second child."

\section{DISCUSSIONS}

\subsection{Family planning policy under the demographic transition context.}

The survey result indicated that the transition of demographic and family structures post threat to the family planning policy.

Similarly, the interview result indicated that the national policy for a fertility will mainly focus on aging population, the decreasing of demographic dividend and the inertia of the one-child policy. The one child policy had a strong inertia to the two-child policy, on the other hand, two-child policy was a good alternative to the onechild policy for solving the remaining social issues but cannot redirect the one-child policy's thought pattern.

\subsubsection{From the one-child policy to the two-child policy: the factors of having the second child.}

The survey result indicated that the half of the participants agreed the two-child policy was a good alternative to the one-child policy. Since the two-child policy has been established for almost six years, the onechild policy still has the impact on individual's mindsight. Some of the non-policy factors played a more direct role on having the second child. Specifically, the interview result indicated that the childhood experience, marital relationship and resources became the common factors which influenced individual's fertility preferences.

\section{CONCLUSION}

The one-child policy was developed with the intent to benefit the Chinese people. The anticipated benefits were expected to allow controlling the population effectively. The second intended benefit was the alleviation of poverty. The high population in China was causing strain in social, economic, and natural resources, thus depleting them to the extent that the next generation would lack them. The third intended benefit that encouraged the development and enactment of one-child policy was the need to improve the country's economy, which was fast declining because of the rapid population growing, which had adverse effects on such areas as the healthcare system and education. Upon implementation, the policy managed to achieve some of the intended goals.

Some of the positive consequences attributable to one-child policy include the increase in the total number of offspring who were able to attain high levels of education. This trend benefited a large number of people because couples who had only one child were able to spend all their resources on their only offspring. Another positive effect was the improved wellbeing of children in China's households, especially those that were made up of low-income earners. The families were able to cater to their child's needs better than they would do had they many children.

The alternative policy, namely the two-child policy, seemingly has a positive effect on China and its people. From the survey and interviews, the two child policy is a good alternative to the one-child policy, because it can solve remaining social problems from one-child policy. But the two child policy is not the key factor of individual birth inclinations. People more care about their personal preference on having the second child which are childhood experience, marital relationship and resources.

\section{REFERENCES}

[1] Albers, Michael, J. "Quantitative DataAnalysis-In the Graduate Curriculum."Journal of Technical Writing and Communication 47, no. 2 (2017): 215 233.

[2] Doepke, Matthias. "Gary Becker on the Quantity and Quality of Children." Journal of Demographic Economics 81, no. 1 (2015): 59-66. 
[3] Falbo, Toni. "Evaluations of the Behavioral Attributes of Only Children in Beijing, China: Moderating Effects of Gender and the One-Child Policy." Heliyon 4, no. 4 (2018): 1-14.

[4] Howden, David, and Yang Zhou. "China's One-Child Policy: Some Unintended Consequences." Economic Affairs 34, no. 3 (2014): 353-369.

[5] Ke, Qing, Li Zhang, Chaying He, Zhengyan Zhao, Ming Qi, and Robert C Griggs. "China's Shift from Population Control to Population Quality: Implications for Neurology." Neurology 87, no. 8 (2016): 85-88.

[6] Liu, Tao-Xiong, and Jun Liu. Two-Child Policy, Gender Income and Fertility Choice in China.Munic, Germany: MPRA, 2017.

[7] Sudbeck, Kristine. "The Effects of China's One-Child Policy: The Significance for Chinese Women." Nebraska Anthropologist 27, no. 179 (2012): 43-60.

[8] Taherdoost, Hamed. "Sampling Methods in Research Methodology; How to Choose a Sampling Technique for Research." SSRN Electronic Journal 5, no. 2 (2016): 18-27.

[9] Zeng, Yi, and Therese Hesketh. "The Effects of China's Universal Two-Child Policy." The Lancet 388, no. 10054 (2016): 19301938. 